\title{
Primary signet ring cell carcinoma of colon and rectum often presents as long segment stenosis in young patients
}

\author{
Meng Tzu Weng ( $\nabla$ wengmengtzu@gmail.com ) \\ National Taiwan University Hospital https://orcid.org/0000-0002-4143-6607 \\ Ko-Han Chao \\ Departments of Internal Medicine \\ Chien-Chih Tung \\ Departments of Integrated Diagnostics and Therapeutics \\ Hao-Chun Chang \\ Departments of Internal Medicine \\ I-Lun Shih \\ Department of Medical Imaging \\ Been-Ren Lin \\ Department of Surgery \\ Ming-Jium Shieh \\ Department of Oncology \\ Chia-Tung Shun \\ Department and Graduate Institute of Forensic Medicine \\ Jau-Min Wong \\ Departments of Internal Medicine \\ Shu-Chen Wei \\ Departments of Internal Medicine
}

\section{Research}

Keywords: Primary signet ring cell carcinoma, colorectal cancer

Posted Date: April 29th, 2020

DOI: https://doi.org/10.21203/rs.3.rs-24978/v1

License: (c) (i) This work is licensed under a Creative Commons Attribution 4.0 International License. Read Full License 


\section{Abstract}

Background: Primary signet ring cell carcinoma of the colon and rectum (PSRCCR) is rare, usually diagnosed at advanced stage with poor outcomes. We aimed to find possible diagnostic clues in order to help diagnosis.

Methods: A retrospective study of PSRCCR patients from 1993 to 2018 was reviewed at a single tertiary center. Colorectal adenocarcinoma patients as control group with 1:4 ratio was also enrolled.

Results: 18 patients with PSRCCR were identified. The prevalence rate was $0.16 \%$ (18 of 11,515$)$. The mean age was 50.2 years-old in PSRCCR group and 63 years-old in non-SRCC colorectal cancer patients $(p<0.001)$. Diagnosis tool depends on CFS were much less in PSRCCR group than control group $(44.4 \%$ vs $93 \%, p<0.001)$. SRCC patients had higher level of CEA (68.3 vs $17.7 \mathrm{ng} / \mathrm{mL}, \mathrm{p}=0.004$ ) and lower level of Albumin (3.4 vs $4.3 \mathrm{~g} / \mathrm{dL}, \mathrm{p}<0.001$ ). The majority of PSRCCR tumor configuration was ulcerative and infiltrative. More PSRCCR pathology presented as high-grade carcinoma (66.7 vs $1.4 \%, p$ $<0.001)$ and lymphovascular invasion (77.8 vs $44.4 \%, p=0.011)$ than control group. More PSRCCR patients were diagnosed at advanced stage (88.8 vs $40.3 \%, p=0.001)$. Higher mortality was also noticed in PSRCCR group than control group (72.2 vs $20.8 \%, \mathrm{p}<0.001)$.

Conclusion: For young patients with long segment colonic stenosis and ulcerative/ infiltrative mucosa but endoscopic biopsy failed to identify malignant cells, earlier operation or non-colon site biopsy is suggested for diagnosing the PSRCCR.

\section{Background}

Primary signet ring cell carcinoma of the colon and rectum (PSRCCR) is a rare histologic subtype, accounting for approximately $0.6-2.7 \%$ of all adenocarcinomas of the colon $(1,2)$. Signet ring cell carcinoma (SRCC) contains a large amount of mucin, which pushes the nucleus to the cell periphery. The World Health Organization has a specific criterion for diagnosing this subtype; that is, the presence of $>50 \%$ of signet cells (3). The symptoms of PSRCCR include bloody stool, body weight loss, abdominal pain or bowel habit change and usually appear at late stage (4). The symptoms are similar to those of colorectal cancer (CRC) (5), whereas the clinical behavior is more aggressive than colorectal cancer. Patient with colonic signet ring cell carcinoma were more frequently diagnosed at advanced stage (75.2 - 91\%) (6-8) than patients with colon adenocarcinoma (43.6 - 48\%). Less than $40 \%$ of cases have a change to receive curative operation (9). Compared with non-SRCC colorectal cancer, PSRCCR tends to occur at a younger age, presented as scirrhous appearance, with more lymphovascular and peritoneal involvement, and has a poorer prognosis $(6,10,11)$.

As PSRCCR is a rare subtype and its characteristic is different from common colon adenocarcinoma. In this study, we aimed to compare the difference of clinical characteristics, pathologic features, diagnostic stage and outcome between patients with SRCC and non- SRCC colorectal cancer. Through this comparison, we also would like to find the possible diagnostic clues in order to help diagnosis.

\section{Methods}

\section{Data Collections}

This study was approved by the Ethics Review Board of National Taiwan University Hospital (IRB Number 201808070RINB). In total, 11,515 colorectal cancer tissues were received and registered in the pathology databank from October 1993 to June 2018 in National Taiwan University Hospital. Clinical information including demographic data, laboratory, endoscopic and pathologic report, treatment regiments and the disease course were assessed. Tumor stage was based on TNM staging system and American Joint Committee on Cancer, AJCC Cancer Staging Manual (8th edition, 2017). Stage I and II were defined as early stage and stage III and IV as advanced stage. The survival duration was based on the 
last outpatient department date or date of death. A retrospective computer-aided search generated 18 PSRCCR cases. We match PSRCCR patients with non-PSRCCR colorectal cancer patients as a control group at a 1: 4 ratio.

\section{Statistical analyses}

Results are expressed as the mean and range. Continuous variables were expressed as mean \pm standard deviation (SD). Categorical variables were expressed as frequency (percentage). Student's t-test was used for quantitative variables and Chi-square statistic was used for categorical variables among the two cohorts. A p-value less than 0.05 was considered as statistically significant. The survival was calculated using the Kaplan-Meier method. Prognostic factors including age, sex, underlying disease, laboratory data, tumor subtype, pathological parameters, cancer stage were included in survival analyses. Parameters with $\mathrm{P}<0.05$ in univariable analyses were further checked by multivariable Cox proportional hazard model. These analyses were carried out with SPSS 11.0 program (SPSS, Paris, France).

\section{Results}

\section{Demographic and clinical characteristics of PSRCCR patients}

A total of 11,515 patients were diagnosed with colorectal cancer from the hospital database between 1993 and 2018 . Among them, 18 were identified with PSRCCR. The incidence of PSRCCR was $0.16 \%$ in colorectal patients. A total of 72 patients with non-SRCC CRC were included as controls. PSRCCR patients was significant younger than non-SRCC CRC patients (mean age 50.2 vs 63 years-old, $p<0.001$ ). In both the PSRCCR and non- SRCC groups, male predominance was noted $(66.7 \%$ vs $62.5 \%, p=0.743)$. The baseline hypertension $(22.2 \%$ vs. $38.9 \%, p=0.186)$, diabetes mellitus ( $5.6 \%$ vs. $16.7 \%, p=0.23$ ), hyperlipidemia ( $0 \%$ vs. $12.5 \%, p=0.195)$, viral hepatitis ( $5.6 \%$ vs. $5.6 \%, p=1)$, coronary artery disease ( $5.6 \%$ vs. $11.1 \%, p=0.482$ ) and chronic kidney disease (5.6 \% v. $9.7 \%, p=0.578$ ), was comparable in these two groups. Most of the non-SRCC CRC patients were diagnosed by colonoscopy. In contrast, more than one-third PSRCCR patients were diagnosed by operation or non-colon site biopsy $(p<0.001)$. The PSRCCR group was associated with higher level of CEA (68.3 vs $17.7 \mathrm{ng} / \mathrm{mL}, p=0.004)$, the albumin level was significantly lower in the PSRCCR group than in the non-SRCC group (3.4 vs $4.3 \mathrm{~g} / \mathrm{dL}, p<0.001$ ). The clinical information of PSRCCR and non-SRCC CRC patients were summarized in Table 1.

\section{Comparison of pathologic characteristics between PSRCCR and non- SRCC CRC patients}

Majority of the tumor were located at left side in both groups $(61.1 \%$ vs $68.1 \%, p=0.586)$. Most tumor configuration of PSRCCR patients were ulcerating or infiltrative, whereas those of non-SRCC patients were exophytic or ulcerating ( $p<$ 0.001). The differentiation grade of PSRCCR group was significantly advanced than that of non-SRCC group (66.7\% vs 1.4 $\%$ high grade, $p<0.001)$. PSRCCR patients also had more lymphovascular invasion than non-SRCC patients (77.8 \% vs 44.4 $\%, p=0.011)$. The pathologic features were listed in Table 2 .

\section{Comparison of tumor stage between PSRCCR and non- SRCC CRC patients}

Most of SRCC patients were diagnosed at stage T3 or T4 (94.4\%) and N2 (77.8\%). The distant metastasis rate was $50 \%$. The only patient who was diagnosed with a tumor at an early stage, with carcinoma in situ, was due to a positive immunochemical fecal occult blood test during health examination. The number of the patients with initial AJCC stages 0 and 1, 2, 3, and 4 was 1 (5.6\%) versus 0 (0\%), 0 (0 \%) versus $23(31.9 \%), 1$ (5.6\%) versus $20(27.8 \%), 9$ (50\%) versus 18 $(25 \%)$ and $7(38.8 \%)$ versus $11(15.3 \%)$ in the PSRCCR and non-SRCC groups, respectively $(p=0.001)$. All these CRC patients underwent operation. As most PSRCCR patients were diagnosed at advanced stage, $88.9 \%$ of them also received chemotherapy or combine therapy. In contrast, a curative resection (R0 resection with related radical lymphatic dissection) was performed in 37 (51.4 \%) non-SRCC patients. The PSRCCR group had shorter follow-up period than non-SRCC group (15 vs 94.5 months, $p<0.001$ ) (Table 3). The clinical characteristic of the 18 PSRCCR patients were listed in Table 4. 
Seventeen PSRCCR patients with initial computed tomography (CT) were reviewed. Most of the patients (14 of 17; $82.3 \%)$ presented with long segmental wall thickening and increased enhancement of the involved colon. The average length of the thickened wall was $6.6 \mathrm{~cm}$ (range: $4.4-11.6 \mathrm{~cm}$ ). Only 2 of the $17(11.8 \%)$ patients presented with an intraluminal mass. One patient (5.9\%) had carcinoma in situ, which could not be identified on CT.

\section{Comparison of survival status between PSRCCR and non- SRCC CRC patients}

The patients in the PSRCCR group had significantly poorer estimated overall survival than those in the Non-SRCC group (29.6 vs 162.7 months, log-rank $p<0.001$, Fig. 1A). After stratification, the patients with PSRCCR had significantly poorer estimated overall survival than did the patients with non-SRCC of early stage CRC ((37 vs. 122.8 months, log-rank $p<0.001$, Fig. 1B) and advanced stage CRC (18 vs. 140.7 months, log-rank $p<0.001$, Fig. 1C). Since majority of the patients were diagnosed at advanced stage, most of them received chemotherapy and target therapy. Target therapy prescribed in this study included Cetuximab and Bevacizumab, and all non-SRCC CRC patients received Bevacizumab. Non-SRCC patients still had better prognosis than PSRCCR patients no matter which target therapy they used (Figure 2A). Target therapy treated or not didn't improve the overall survival of PSRCCR patients (Figure 2B and C).

\section{Factors associated with overall survival}

In the univariable analysis of overall survival in all CRC patients, poor differentiation grade, lymphovascular invasion, advanced cancer stage, high CEA level and histological SRCC subtype were associated with increased mortality rates (all $p$ $<0.05$, Table 4). Further multivariate analysis with adjusted Cox proportional hazard model revealed that the CEA level (HR, $1.003 ; 95 \% \mathrm{Cl}, 1.000-1.005 ; p=0.03)$ and histological SRCC subtype (HR, 8.333; 95\% Cl, 1.42-50; $p=0.005)$ were independent predictors of overall survival. The detail information of univariable and multivariate factor were listed in Table 5 .

\section{Discussion}

Primary signet ring cell carcinoma of colon and rectum is a rare variant of CRC. The frequency of SRCC was no difference between Western and Eastern Countries. In this study, 18 of the 11,515 CRC patients were diagnosed with PSRCCR. It accounted for $0.16 \%$ of all primary CRCs. The percentage of PSRCCR was even lower in our study than those of previous studies which indicated $0.6 \%-2.7 \%(1,7,10-12)$.

Compared to non-SRCC patients, PSRCCR patients were younger. In Wang's study, they also reported PSRCCR is four times more prevalent among the young (ages $<40$ years) than older adults ( $>40$ years) (13). The underlying disease including hypertension, diabetes mellitus, viral hepatitis, coronary artery disease and chronic kidney disease were not significant different between PSRCCR and non-SRCC patients. Most (88.8\%) PSRCCR patients were diagnosed at advanced stage as their initial presentations were non-specific such as abdominal fullness, pain and bowel habit change. The PSRCCR patients were also associated with low level of albumin and higher CEA level than non-SRCC patients.

Most of our patients' CT finding (82.3\%) presented with long segmental wall thickening rather than an intraluminal mass. In Kim's study, they also reported the CT features of PSRCCR was long segmental $(>5 \mathrm{~cm})$ concentric bowel wall thickening without an intraluminal mass, which resembles the inflammatory process (2). $82.4 \%$ of tumor configuration were ulcerative or infiltrative type and only $17.6 \%$ were exophytic type in the PSRCCR group. However, in non-SRCC CRC group, infiltrative type only accounted for $4.2 \%$ and exophytic type for $47.2 \%$. This is compatible with Messerini study ${ }^{7}$ that revealed that infiltrative type was predominant in PSRCCR tumors (70.6\% infiltrative type and $29.4 \%$ exophytic type). Most colorectal cancer cases (93.1\%) were diagnosed by colonoscopic biopsy in control group, whereas 4 of SRCC patients (22.2\%) ever received colonoscopic biopsy but failed to identify malignant cells. As $82 \%$ of PSRCCR were infiltrative or ulcerative type, it increased the difficulty to identify cancer cell by endoscopic biopsy and led to delay diagnosis. This also explained the reason that half of PSRCCR patients were diagnosed depended on direct operation for colon obstruction, bloody stool or 
malignant cells identified at non-colon site. Long segment colonic stenosis in young patients, also leading to the possibility of inflammatory bowel disease. Indeed, at least one of this PSRCCR cohort was treated as Crohn's disease initially. Therefore, close monitoring the treatment effect and get histology diagnosis are important in the differential diagnosis.

Half of non-SRCC CRC patients underwent curative operation and had good prognosis. Most of PSRCCR patients were diagnosed at advanced stage and received combination therapy, whereas chemotherapy or target therapy both failed to improve survival. We also stratified and analyzed the treatment efficacy of bevacizumab or Cetuximab and showed poorer response in SRCC patients. Previously, SRCC has been shown to have fair response to chemotherapy and our results also confirmed this result. Furthermore, we showed that even with current available target therapy (anti- vascular endothelial growth factor and/or anti- epidermal growth factor receptor), the survival still could not be improved. Novel therapy, either chemotherapy or target therapy for PSRCCR patients, remains as an unmet need.

The estimated overall survival time of the PSRCCR patients (26.9 months) was much shorter than that of non-SRCC patients (162.7 months). SRCC subtype and elevated CEA were independent predictors of overall survival by using Cox proportional hazard regression model. Compared with non-SRCC patients, PSRCCR patients had higher risk of lymphovascular invasion, poor-differentiated carcinoma, visceral peritoneum invasion, lymph node and distant metastasis. This also indicated that the behavior of PSRCCR was more aggressive. According to Huang et al ${ }^{(14)}$ study, patients aged $<35$ years had shorter cancer-specific survival compared with those aged $>35$ years, and the 5 -year cancer-specific survival rates were $31.1 \%$ and $54.9 \%$ in patients aged $\leq 35$ and $>35$ years, respectively. Five of the 18 patients in our series were aged $<35$ years. The mean survival time was 20.6 months in this group. The survival time was not significantly different between these two groups. However, the results should be interpreted cautiously because of relatively few cases.

There were still limitations of this study. First, this was a retrospective observable study in single referral center. Second, the case number was small. As the PSRCCR is a very rare disease, it is difficult to collect many patients or perform a prospective study.

\section{Conclusion}

Primary signet ring cell carcinoma of colon and rectum usually present as infiltrative or ulcerative configuration and is associated with poor differentiation, higher lymphovascular invasion and distant metastasis. Signet ring cell carcinoma is a strong predictor of poor overall survival. For young patients with colonic long segment stenosis and ulcerative/ infiltrative mucosa but endoscopic biopsy failed to identify malignant cells, PSRCC should be considered. Even with the progress in current chemotherapy and target therapy, they seemed to be with limited effect in improving the survial of PSRCCR patients and we still need to work out the novel therapy in order to improve the outcomes of these patients.

\section{Abbreviations}

CRC: Colorectal cancer

CT: Computed tomography

PSRCCR: Primary signet ring cell carcinoma of the colon and rectum

SRCC: Signet ring cell carcinoma

\section{Declarations}

Ethics approval and consent to participate: This study was approved by the Ethics Review Board of National Taiwan University Hospital (IRB Number 201808070RINB) 
Consent for publication: Not applicable

Availability of data and materials: The datasets used and/or analysed during the current study are available from the corresponding author on reasonable request.

Competing interests: The authors declare that they have no competing interests

Funding: This research was supported by the National Taiwan University Hospital (NTUH 107-A139 to S.-C.W.) the Liver Disease Prevention and Treatment Research Foundation, Taiwan (to M.-T.W.).

Authors' contributions: M.-T.W., K.-H.C., and I.-L.S: drafting of the manuscript; C.-C.T., C.-T.S. and S.-C.W.: analysis and interpretation of the data; K.-H.C. and H.-C.C., and B.-R.L.: acquisition of the data; M.-T.W.: statistical analysis; M.-J.S., J.M.W., and S.-C.W.: critical revision of the manuscript for important intellectual content and study supervision; and S.-C.W. and J.-M.W.: study concept and design.

Acknowledgements: Not applicable.

\section{References}

1. Thota R, Fang X, Subbiah S. Clinicopathological features and survival outcomes of primary signet ring cell and mucinous adenocarcinoma of colon: retrospective analysis of VACCR database. J Gastrointest Oncol. 2014;5(1):18-24.

2. Kim HJ, Ha HK, Cho KS, Yu E, Kim JC, Yoo CS, et al. CT features of primary colorectal signet-ring cell carcinoma. J Comput Assist Tomogr. 2001;25(2):225-30.

3. SR. H LA. World Health Organization classification of tumours. Pathology and Genetics of Tumours of the digestive system: IARC Press; 2000.

4. Tung SY, Wu CS, Chen PC. Primary signet ring cell carcinoma of colorectum: an age- and sex-matched controlled study. Am J Gastroenterol. 1996;91(10):2195-9.

5. Farraj FA, Sabbagh H, Aridi T, Fakhruddin N, Farhat F. Signet Ring Cell Carcinoma of the Colon in Young Adults: A Case Report and Literature Review. Case Rep Oncol Med. 2019;2019:3092674.

6. Nitsche U, Zimmermann A, Spath C, Muller T, Maak M, Schuster T, et al. Mucinous and signet-ring cell colorectal cancers differ from classical adenocarcinomas in tumor biology and prognosis. Ann Surg. 2013;258(5):775-82; discussion 82-3.

7. Belli S, Aytac HO, Karagulle E, Yabanoglu H, Kayaselcuk F, Yildirim S. Outcomes of surgical treatment of primary signet ring cell carcinoma of the colon and rectum: 22 cases reviewed with literature. Int Surg. 2014;99(6):691-8.

8. Hugen N, Verhoeven RH, Lemmens VE, van Aart CJ, Elferink MA, Radema SA, et al. Colorectal signet-ring cell carcinoma: benefit from adjuvant chemotherapy but a poor prognostic factor. Int J Cancer. 2015;136(2):333-9.

9. Radhakrishnan CN, Bruce J. Colorectal cancers in children without any predisposing factors. A report of eight cases and review of the literature. Eur J Pediatr Surg. 2003;13(1):66-8.

10. Kang H, O'Connell JB, Maggard MA, Sack J, Ko CY. A 10-year outcomes evaluation of mucinous and signet-ring cell carcinoma of the colon and rectum. Dis Colon Rectum. 2005;48(6):1161-8.

11. Sasaki S, Masaki T, Umetani N, Futakawa N, Ando H, Muto T. Characteristics in primary signet-ring cell carcinoma of the colorectum, from clinicopathological observations. Jpn J Clin Oncol. 1998;28(3):202-6.

12. Messerini L, Palomba A, Zampi G. Primary signet-ring cell carcinoma of the colon and rectum. Dis Colon Rectum. 1995;38(11):1189-92.

13. Wang R, Wang MJ, Ping J. Clinicopathological Features and Survival Outcomes of Colorectal Cancer in Young Versus Elderly: A Population-Based Cohort Study of SEER 9 Registries Data (1988-2011). Medicine (Baltimore). 2015;94(35):e1402. 
14. Huang B, Ni M, Chen C, Feng Y, Cai S. Younger Age Is Associated with Poorer Survival in Patients with Signet-Ring Cell Carcinoma of the Colon without Distant Metastasis. Gastroenterol Res Pract. 2016;2016:2913493.

\section{Tables}

Table 1. Clinical characteristics of colorectal cancer patients

\begin{tabular}{|cccc|}
\hline Demographics & SRCC (n=18) & Non-SRCC (n=72) & $p$-value \\
Age, mean (25-75 th) & $50.2(29.5-67.5)$ & $63.0(54.5-72.5)$ & $p<0.001$ \\
Male sex (\%) & $12(66.7)$ & $45(62.5)$ & $p=0.743$ \\
Underlying diseases, n (\%) & & & \\
Hypertension & $4(22.2)$ & $28(38.9)$ & $p=0.186$ \\
Diabetes Mellitus & $1(5.6)$ & $12(16.7)$ & $p=0.23$ \\
Hyperlipidemia & $0(0)$ & $9(12.5)$ & $p=0.195$ \\
Viral hepatitis & $1(5.6)$ & $4(5.6)$ & $p=1$ \\
Coronary artery disease & $1(5.6)$ & $8(11.1)$ & $p=0.482$ \\
Chronic kidney disease & $1(5.6)$ & $7(9.7)$ & $p=0.578$ \\
Diagnosis tool & $9(50)$ & $67(93.1)$ & $p<0.001$ \\
CFS & $9(50)$ & $5(6.9)$ & \\
Operation or non-colon biopsy & & & \\
Laboratory & 68.3 & 73.7 & $p=0.004$ \\
CEA (ng/mL) & 7485 & 12.3 & $p=0.829$ \\
WBC (K/ $/ \mu$ L) & 12.3 & 4.3 & $p=0.258$ \\
Hb (g/dL) & 3.4 & 25.3 & $p=0.001$ \\
Alb (g/dL) & 20.4 & 1.07 & $p=0.414$ \\
ALT (U/L) & 0.88 & & \\
Cre (mg/dL) & & & \\
\hline
\end{tabular}

CEA: Carcinoembryonic antigen. WBC: White blood cell; Hb: Hemoglobin; Alb: Albumin; ALT, alanine aminotransferase; Cre: Creatinine.

Student's t-test was used for quantitative variables and Chi-square statistic was used for categorical variables among the two cohorts.

Table 2. Pathologic characteristics of colorectal cancer patients

\begin{tabular}{|cccc|}
\hline Tumor location & SRCC (n=18) & Non-SRCC (n=72) & $p$-value \\
Right side & & & $p=0.586$ \\
Left side & $7(38.9)$ & $23(31.9)$ & \\
Tumor configuration & $11(61.1)$ & $49(68.1)$ & $p<0.001$ \\
Exophytic & $3(17.6)$ & $34(47.2)$ & \\
Ulcerating & $7(41.2)$ & $35(48.6)$ & \\
infiltrative & $7(41.2)$ & $3(4.2)$ & $p<0.001$ \\
Grade & $1(5.6)$ & $69(95.8)$ & \\
Low & $12(66.7)$ & $1(1.4)$ & \\
High & $5(27.7)$ & $2(2.8)$ & $p=0.011$ \\
Non-applicable & $14(77.8)$ & $32(44.4)$ & $p=0.597$ \\
Lymphovascular invasion & $1(5.6)$ & $3(4.2)$ & $p$ \\
Surgical margin involvement & & &
\end{tabular}

Chi-square statistic was used for categorical variables among the two cohorts.

Table 3. Treatment and tumor stage of colorectal cancer patients 


\begin{tabular}{|c|c|c|c|}
\hline Treatment & SRCC $(n=18)$ & Non-SRCC $(n=72)$ & $p$-value \\
\hline $\begin{array}{c}\text { Operation } \\
\text { Op }+ \text { C } / \mathrm{T} \\
\text { Op }+\mathrm{C} / \mathrm{T}+\mathrm{Target} \\
\text { Op }+ \text { CCRT }\end{array}$ & $\begin{array}{c}2(11.1) \\
4(22.2) \\
10(55.6) \\
2(11.1)\end{array}$ & $\begin{array}{c}37(51.4) \\
20(27.8) \\
11(15.3) \\
4(5.6)\end{array}$ & $p=0.001$ \\
\hline $\begin{array}{l}\text { Tis } \\
1 \\
2 \\
3 \\
4\end{array}$ & $\begin{array}{c}1(5.6) \\
0 \\
0 \\
8(44.4) \\
9(50)\end{array}$ & $\begin{array}{c}0 \\
4(5.6) \\
22(30.5) \\
36(50) \\
10(13.9)\end{array}$ & $=0.002$ \\
\hline $\begin{array}{l}0 \\
1 \\
2\end{array}$ & $\begin{array}{l}2(11.1) \\
2(11.1) \\
14(77.8)\end{array}$ & $\begin{array}{cc}44 & (61.1) \\
21 & (29.2) \\
7 & (9.7)\end{array}$ & 0.001 \\
\hline $\begin{array}{l}0 \\
1\end{array}$ & $\begin{array}{ll}9 & (50) \\
9 & (50)\end{array}$ & $\begin{array}{l}62(86.1) \\
10(13.9)\end{array}$ & $\rho=0.00$ \\
\hline $\begin{array}{l}\text { Stage } \\
1 \\
1 \\
2 \\
3 \\
4 \\
\text { Follow-up duration, mean (months) }\end{array}$ & $\begin{array}{l}1(5.6) \\
0 \\
1(5.6) \\
9(50) \\
7(38.8) \\
\quad 15\end{array}$ & $\begin{array}{l}0 \\
23(31.9) \\
20(27.8) \\
18(25) \\
11(15.3) \\
94.5\end{array}$ & $p<0.001$ \\
\hline
\end{tabular}

Op: operation; CT: Chemotherapy; CCRT: concurrent chemoradiotherapy.

Chi-square statistic was used for categorical variables among the two cohorts.

Table 4. Clinical characteristics of SRCC patients 


\begin{tabular}{|c|c|c|c|c|c|c|c|c|}
\hline No. & Age & Sex & Main symptoms & CT Findings & $\begin{array}{l}\text { Diagnosis } \\
\text { tool }\end{array}$ & $\begin{array}{l}\text { Cancer } \\
\text { stage }\end{array}$ & $\begin{array}{l}\text { FU } \\
\text { duration } \\
\text { (m) }\end{array}$ & Survival \\
\hline 1 & 36 & $\mathrm{M}$ & Abdominal fullness & $\begin{array}{l}\text { Bowel wall } \\
\text { thickening with }\end{array}$ & CFS & T4bN2bM1c & 9 & Loss FU \\
\hline 2 & 74 & M & Bloody stool & $\begin{array}{l}\text { proximal dilatation } \\
\text { Bowel wall } \\
\text { thickening with }\end{array}$ & CFS & T4aN2bM1c & 23 & no \\
\hline 3 & 86 & $\mathrm{~F}$ & Colon obstruction & $\begin{array}{l}\text { serosal invasion } \\
\text { Bowel wall } \\
\text { thickening }\end{array}$ & CFS & T4aN2bM1a & 1 & Loss FU \\
\hline 4 & 28 & $\mathrm{~F}$ & Bloody stool & NS & CFS & T3N2bM0 & 13 & no \\
\hline 5 & 45 & $\mathrm{~F}$ & Incidental finding & $\begin{array}{l}\text { Semi- } \\
\text { circumferential }\end{array}$ & Operation & $\mathrm{T} 4 \mathrm{~N} 2 \mathrm{bM} 0$ & 19 & Loss FU \\
\hline 6 & 14 & M & $\begin{array}{l}\text { hemorrhoidectomy } \\
\text { Chronic diarrhea }\end{array}$ & $\begin{array}{l}\text { mass } \\
\text { Bowel wall } \\
\text { thickening and }\end{array}$ & CFS & T4bN2bM1c & 17 & no \\
\hline 7 & 17 & M & Bloody stool & Bowel wall & Skin & T3N2bM1b & 14 & no \\
\hline 8 & 76 & M & Bloody stool & Bowel wall & Operation & T3N0M0 & 37 & no \\
\hline 9 & 61 & M & $\begin{array}{l}\text { Positive stool } \\
\text { occult blood }\end{array}$ & Negative finding & CFS & TisN0M0 & 100 & yes \\
\hline 10 & 31 & M & Abdominal fullness & $\begin{array}{l}\text { Bowel wall } \\
\text { thickening }\end{array}$ & Operation & T4aN2bM1c & 44 & Loss FU \\
\hline 11 & 76 & M & $\begin{array}{l}\text { Right lower limb } \\
\text { edema }\end{array}$ & $\begin{array}{l}\text { Bowel wall } \\
\text { thickening }\end{array}$ & $\begin{array}{l}\text { Abdominal } \\
\text { LN biopsy }\end{array}$ & T3N2bM0 & 15 & no \\
\hline 12 & 78 & M & Constipation & Bowel wall & CFS & T3N2bM0 & 8 & no \\
\hline 13 & 40 & $\mathrm{~F}$ & Abdominal fullness & $\begin{array}{l}\text { thickening } \\
\text { Bowel wall } \\
\text { thickening and } \\
\text { regional LAP }\end{array}$ & CFS & T4bN2bM1c & 10 & no \\
\hline 14 & 39 & M & Anemia & $\begin{array}{l}\text { Bowel wall } \\
\text { thickening }\end{array}$ & $\begin{array}{l}\text { Peritoneal } \\
\text { biopsy }\end{array}$ & T3N2aM1C & 14 & no \\
\hline 15 & 57 & $\mathrm{~F}$ & Anemia & $\begin{array}{l}\text { Bowel wall } \\
\text { thickening and } \\
\text { regional LAP }\end{array}$ & CFS & T3N2bM0 & 17 & no \\
\hline 16 & 85 & M & Colon perforation & $\begin{array}{l}\text { Bowel wall } \\
\text { thickening and } \\
\text { pneumoperitoneum }\end{array}$ & Operation & T4aN1bM0 & 2 & no \\
\hline 17 & 43 & M & $\begin{array}{l}\text { Incidental finding } \\
\text { by CT }\end{array}$ & Intraluminal mass & Operation & T3N1bM0 & 28 & no \\
\hline 18 & 18 & $\mathrm{~F}$ & Colon obstruction & $\begin{array}{l}\text { Bowel wall } \\
\text { thickening and } \\
\text { abscess }\end{array}$ & Operation & T4aN2bM1c & 15 & no \\
\hline
\end{tabular}

CT: Computed Tomography; LAP: Lymphadenopathy; FU: follow-up; m: month.

Table 5. Univariable and Multivariable Analysis of Overall Survival in colorectal cancer patients.

\begin{tabular}{lcccc}
\hline & \multicolumn{2}{c}{ Univariable Analysis } & \multicolumn{2}{c}{ Multivariable Analysis } \\
\hline & HR (95\% CI) & $P$ value & HR (95\% CI) & $P$ value \\
\hline Age (male vs female) & $0.993(0.969-1.018)$ & 0.574 & & \\
Sex (ma (low vs high) & $1.091(0.49-2.428)$ & 0.832 & & \\
Grade & $0.131(0.057$ vs 0.301) & $<0.001$ & $0.998(0.184-5.4)$ & 0.998 \\
Location (right vs left) & $0.606(0.281-1.306)$ & 0.201 & & \\
Lymphovascular invasion (no vs yes) & $0.265(0.112-0.631)$ & 0.003 & $0.359(0.109-1.186)$ & 0.093 \\
Stage (early vs advanced) & $0.194(0.078-0.484)$ & $<0.001$ & $0.599(0.184-1.947)$ & 0.394 \\
CEA (ng/mL) & $1.003(1.002-1.005)$ & $<0.001$ & $1.003(1.000-1.005)$ & 0.03 \\
Subtype (SRCC vs non-SRCC) & $10.64(4.74-23.8)$ & $<0.001$ & $8.333(1.42-50)$ & 0.005 \\
\hline
\end{tabular}

The multivariate analysis only included the variables which $\mathrm{P}$ value $<0.05$ in univariate analysis.

HR: Hazard ratio, CI: confidence interval. 
A.

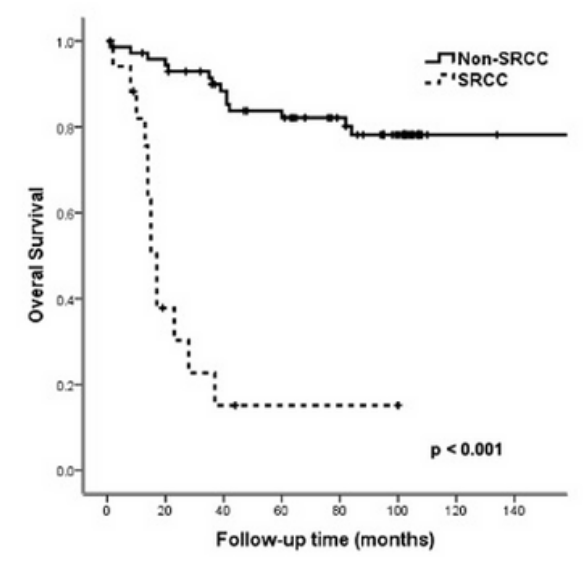

B.

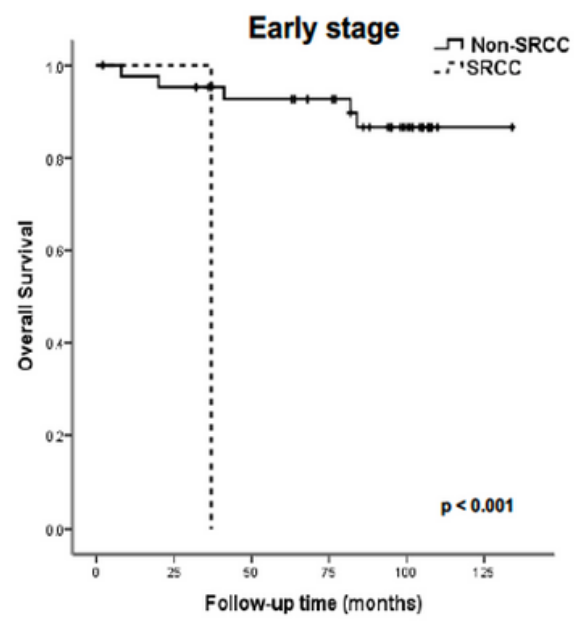

C.

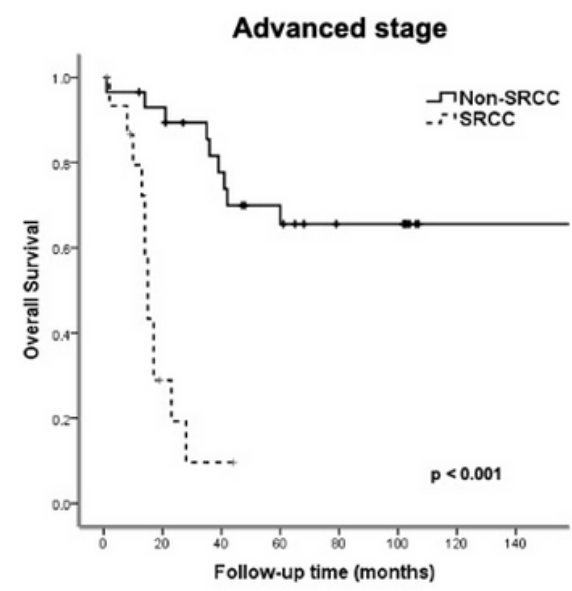

\section{Figure 1}

Kaplan-Meier estimated overall survival curves. (A) Overall survival of all patients with SRCC and Non-SRCC CRC. SRCC is associated with poor overall survival (log-rank $P<0.001)$. (B) Overall survival of patients with early stage SRCC and NonSRCC CRC (log-rank P < 0.001). (C) Overall survival of patients with advanced stage SRCC and Non-SRCC CRC. (log-rank P $<0.001)$.

\section{A}

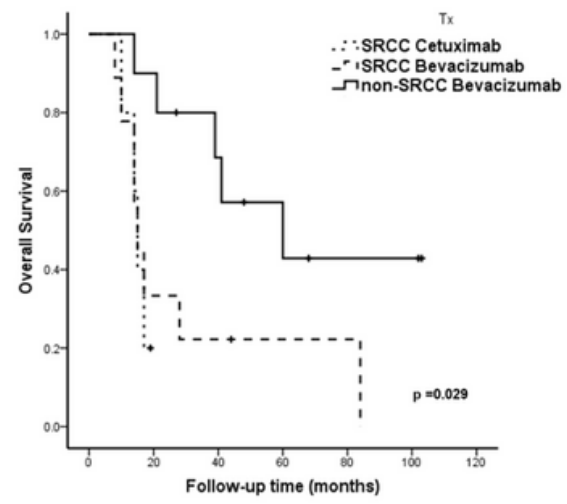

B

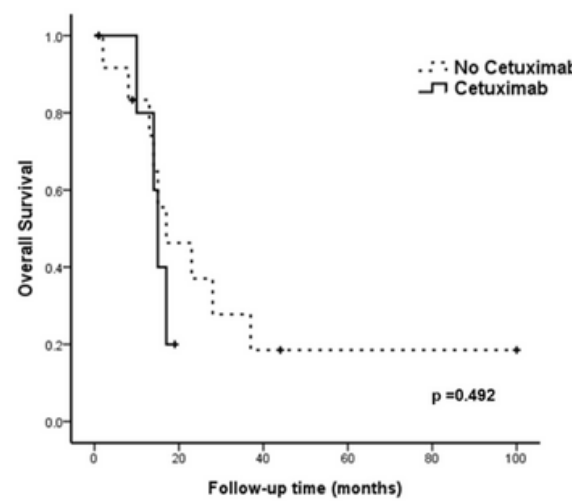

C

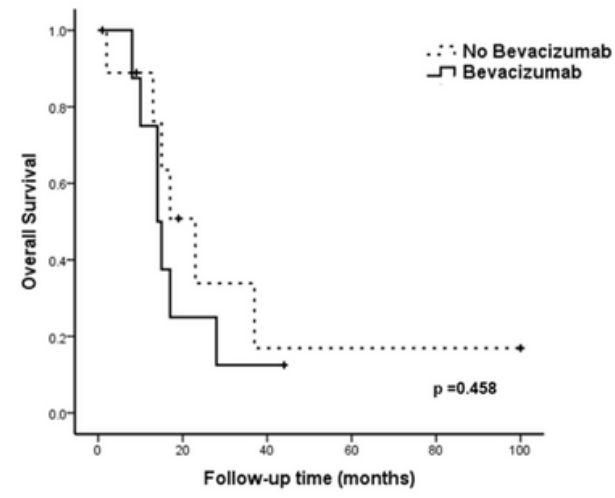

Figure 3

Kaplan-Meier estimated overall survival curves. (A) Overall survival of target therapy treated CRC patients. (B) Overall survival of SRCC patients received Ceuximab or not. (C) Overall survival of SRCC patients received Bevacizumab or not (logrank $\mathrm{P}<0.001)$. 